\title{
Use of the hand held video camcorder in the evaluation of seizures
}

\author{
M Samuel, J S Duncan
}

\begin{abstract}
Epilepsy is primarily a clinical diagnosis and eye witness accounts are invaluable in the differential diagnosis of seizures, but may be incomplete or misleading. A hand held video camcorder was used in this study to record seizures of patients to clarify the nature of their attacks. The videotapes assisted the diagnosis of nonepileptic attacks in nine of 22 patients (41\%), and of epileptic attacks in eight of 22 patients $(36 \%)$. Interactions with patients during filming were particularly helpful. Five patients $(23 \%)$ could not be filmed, three of whom had seizures that were too shortlived to be recorded. In two patients (9\%) a confident diagnosis of non-epileptic seizures could be made from the videotapes alone. Seven patients subsequently required videoEEG telemetry. It is concluded that the hand held video camera is a useful and inexpensive tool to provide accurate seizure descriptions, but it should be used in conjunction with other evidence to classify seizures.
\end{abstract}

(F Neurol Neurosurg Psychiatry 1994;57:1417-1418)

The inpatient assessment unit of the National Hospital for Neurology and Neurosurgery and the National Society of Epilepsy at Chalfont receives tertiary referrals of patients with epilepsy from all over the United Kingdom and in $15 \%$ of patients admitted, non-epileptic seizures are the predominant or sole type of episode experienced by the patient. For the past year, the unit has used a hand held colour video camcorder to record seizures of patients admitted for clarification of diagnosis. The videotapes are reviewed by medical staff as an aid to the classification of seizures. We report the experience with 22 consecutively filmed patients.

National Hospital for Neurology and Neurosurgery, London and National Society for Epilepsy, Chalfont, UK

M Samuel

J S Duncan

Correspondence to:

Dr J S Duncan, National

Hospital for Neurology and

Neurosurgery Queen Square,

London WCIN 3BG, UK.

Received 23 December 1993 and in revised form

18 April 1994.

Accepted 27 April 1994 aged 18 to 55 years, were admitted to the assessment unit for review of diagnosis over a 10 week period. All patients gave written consent to filming.

The camera used was a lightweight, portable, battery operated video camcorder (Panasonic), measuring $15 \times 8 \times 8 \mathrm{~cm}$ and costing about $£ 700$. It had easy operating instructions and was readily available on the ward, allowing rapid employment by the nursing staff. The aim of filming was initially to provide a global picture of the patient as soon as possible after the onset of the seizure, then to utilise the camera's zoom facility to concentrate on certain details - eyes, mouth, presence of cyanosis, posture, and areas of movement. Sound recording enabled breathing patterns and speech to be evaluated. The camera was able to work at levels of illumination down to 3 lux, thus allowing nocturnal seizures to be filmed without the necessity of additional lighting.

During the filming of seizures one member of staff was requested to interact with the patient. Such interactions included response to commands, menace, pain and plantar reflexes, and attempts to open the eyes to assess resistance and direction of gaze. At the end of the seizure, recording was continued for a few minutes to assess speed of recovery as well as other signs that became apparent as patients believed that filming had ceased. All videotapes were subsequently reviewed by the medical and nursing staff.

As part of their continuing assessment, serum prolactin concentrations were measured after convulsive seizures, and all patients underwent routine interictal EEG and a period of continuous EEG monitoring, either by eight channel ambulatory EEG or video-EEG telemetry.

The final classification of seizures was based on the combined evidence of the history, videotapes, EEG recordings, and prolactin estimations.

\section{Results}

It was possible to film 20 seizures from 17 patients $(77 \%)$, as some patients had more than one seizure type. Five patients $(23 \%)$ could not be recorded; two patients (9\%) had no seizures during their stay; and three patients (14\%) had brief absences, myoclonus, or drop attacks that occurred too infrequently and were too brief to allow recording to commence.

Of the 17 patients filmed, the camcorder Twenty two patients (12 women, 10 men),

assisted in the diagnosis of non-epileptic attacks in nine patients ( $41 \%)$ : six patients $(27 \%)$ had non-epileptic attacks only, two patients $(9 \%)$ had both epileptic and nonepileptic attacks, and one patient (5\%) had two types of non-epileptic seizures. In eight patients $(36 \%)$ epilepsy was confirmed and the seizure type classified. 
Ten of the 22 patients (46\%) required video-EEG telemetry. These included the three patients $(14 \%)$ in whom it was impossible to record seizures with the camcorder, and seven patients $(32 \%)$ in whom the camcorder was used but the cumulative evidence to that point had not allowed a confident diagnosis to be reached. Two patients $(9 \%)$ were filmed during a period of 24 hour ambulatory EEG monitoring, thus also providing simultaneous clinical and electroencephalographic evidence.

The presence of cyanosis, grunting, fixed eye deviation, and a tonic phase followed by a clonic phase made four generalised tonicclonic seizures relatively easy to diagnose from the videotapes. In six patients with complex partial seizures, fixed eye deviation to either side or into the distance, associated with automatisms such as dancing in circles, lip chewing, facial slapping, and head or arm posturing were filmed.

In the 10 non-epileptic attacks several features were filmed: avoidance of eye contact or resistance to attempted eye opening (eight), elaborate and prolonged rigidity, opisthotonus, arm waving or leg kicking (seven), facial grimacing to menace or to testing plantar reflexes (four), opening of old wounds and carpet burns (three), voluntary salivation (two), a pronounced difference between epileptic and non-epileptic seizures (two), patients directly interacting with the camera on realising that they were being filmed (two), and self restraint with the arms during falling (one).

\section{Discussion}

The hand held video camcorder can provide an accurate and well documented clinical picture of a seizure, which is often clearer and sometimes different from that which is obtained from an eye witness account. Terms such as "convulsions", "jerks", "thrashing", and "rolling" are ill defined and even experienced medical and nursing staff can have difficulty in describing seizures.

The videotapes provide an opportunity to assess the exact motor sequences involved during a seizure and can clearly document the differences between a generalised tonic-clonic seizure, and one manifested by prolonged and elaborate posturing. This is particularly informative in patients suspected of experiencing both epileptic and non-epileptic attacks. Nonepileptic seizures are most difficult to diagnose in patients with epilepsy, as they may occur in the setting of an abnormal background EEG. ${ }^{12}$ In each of two patients in our study, the videotapes documented the different seizure types, despite the initial witness's descriptions suggesting that each patient had only single types of seizures.

The zoom facility also allowed detailed views of eye position, cyanosis, and purposeful tongue and laryngeal movements to be obtained. Rapid self restraining manoeuvres were well characterised by the slow motion replay facility. Interaction with the patients during seizures, coupled with close filming of the face, allowed us to document a variety of individual signs which suggested that alertness was preserved during a specific seizure, and therefore supported the diagnosis of nonepileptic seizures. It was also valuable to continue recording, especially the face, well after the patient believed that filming had ceased. A quick glance at the camera or the acceptance of "being caught in the act" made two patients' inconsistent alertness obvious.

It should be noted that some complex partial seizures may appear bizarre, ${ }^{34}$ and interpretation even by experienced observers may not be straightforward. Hence, the videotaped evidence must be assessed in conjunction with the history, EEG evidence, and prolactin estimations.

Scalp video-EEG telemetry remains the most useful single diagnostic aid, and simply filming the seizures without concurrent EEG data is rarely definitive. Forty one per cent of our patients subsequently had video-EEG telemetry. The population studied, however, comprised those with difficult diagnostic problems and they were all tertiary referrals. It is possible that simple video recording of seizures would provide a higher yield of diagnostic data in a less highly selected group of patients.

Video-EEG telemetry also has the advantage of being able to capture the onset of seizures at a time that is usually missed by the video camcorder because of the inherent delay, albeit seconds, to initiating filming. For the same reasons video-EEG telemetry can capture brief and unpredictable events (for instance, absences and myoclonic and atonic seizures). The camcorder does, however, have some advantages over videotelemetry: (1) the equipment is cheap, readily available, robust and easy to use; (2) the zoom facility allows detailed views of the eyes, mouth, and face to be obtained; (3) it is possible to track the patient who is moving out of range of a fixed camera; (4) it is easier to record interaction between an observer and the patient.

The video camcorder may also be used in conjunction with ambulatory EEG to provide simultaneous clinical and electroencephalographic information from a seizure, provided accurate timing is recorded. In these circumstances their combined use can obviate the need for videotelemetry.

In conclusion, a hand held video camcorder can be a useful adjunct in the clinical assessment of seizures, as it is accurate, inexpensive, and convenient. The data obtained should be used in conjunction with EEG and other information and, in some cases, may obviate the need for video-EEG telemetry. The videotapes may also be used to educate both patients and professionals about the nature and management of their condition.

We are grateful to the nursing and care staff for their skilled cinemator video-EEG records.

1 Lelliot PT, Fenwick P. Cerebral pathology in pseudoseizures. Acta Neurol Scand 1991;83:129-32.

2 Betts TA Neuropsychiatry. In: Laidlaw J, Richens A Chadwick D, eds. $A$ textbook of epilepsy. 4th ed. Chadwick D, eds. A lextbook 1993.397-457.

Cdinorgent motor seizures mimicking pseudoseizures. Neurology 1990;40:1404-7.

4 Morris HH III, Dinner DS, Lueders H, Wyllie E, Krammer R. Supplementary motor seizures: clinical and Krammer R. Supplementary motor seizures: clinical and
electroencephalographic findings. Neurology 1988;38: electroen 